\title{
ABSORPTION-DELAY MODELS OF HEAT TRANSPORT
}

\author{
R.E. SHOWALTER AND D.B. VISARRAGA
}

\begin{abstract}
A temperature jump in the water traveling through a pipe is delayed by the absorption of heat into the pipe wall. The convective transport of heat by the water and the conductive exchange with the interior pipe wall are described by a singular micromodel. The limiting form is a distributed microstructure model, which is successively better approximated by simpler first- and second-order kinetic models. This identification is used to validate and calibrate these classical multiple-temperature models which describe the delay.
\end{abstract}

\section{Contents}

1. Introduction 2

2. The Singular Micro-Model 3

3. The Distributed Microstructure Model 6

4. Kinetic Models 10

5. Numerical Results 11

References $\quad 15$

Date: January, 2002.

1991 Mathematics Subject Classification. Primary 35K50, 35B25; Secondary 80A20, 35F15.

Key words and phrases. Mathematical modeling, heat transfer, absorption, memory, kinetic models, approximation. 


\section{INTRODUCTION}

Consider a long, circular pipe of length $L$ and radius $\varepsilon>0$, with $\varepsilon<<L$. We assume this pipe is filled with stationary water, and the initial temperature of both the pipe and water are equal to a constant ambient temperature $T_{a m b}$. The outside of the pipe is perfectly insulated throughout its length, the interior pipe wall and water are in perfect thermal contact, and a source of hot water at a fixed temperature $T_{0}>T_{a m b}$ is attached to the left end of the pipe (see Figure 1 ). At time $t=0$, water begins to flow from the hot

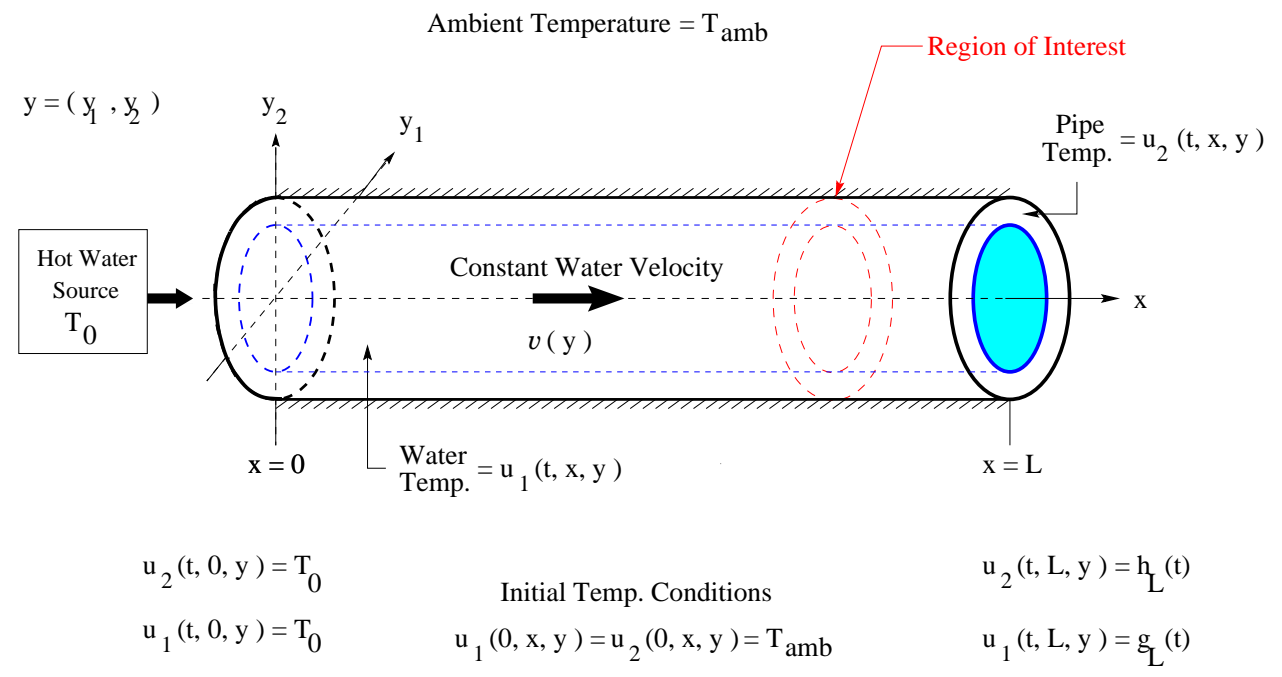

FiguRE 1. Thin-walled circular pipe of length $L$ and radius $\varepsilon$.

water source through the pipe, and we assume this flow is laminar and hydrodynamically fully developed (that is, the velocity of the water $v$ is constant in the direction parallel to its flow). The flushing time $t_{f}(=L / v)$ is the expected minimum time it takes hot water from the source to reach the end of the pipe. Due to the absorption of heat by the pipe, an additional delay occurs in the arrival of hot water. Our objective is to develop an accurate model of this process.

We start with the system of partial differential equations that describes the exact micro-model for the heat transport and heat exchange between the water and interior pipe wall. This system contains two sources of singularity: a geometric one due to the thinness of the pipe with respect to its length, and a material one due to the different conduction properties of the two materials involved, i.e., the pipe and the water. By choosing an appropriate scale between these thermal conductivity values, we find a limiting form of this highly singular micro-model that is fully coupled and handles both of these singularities by properly balancing them. This is a distributed microstructure model consisting of a family of partial differential equations. This parabolic system is shown to be well approximated by a single parabolic equation coupled to a diagonal system of ordinary differential equations. These are directly related to the considerably simpler kinetic models, and thereby we obtain the validation of these classical multi-temperature models and the identification of their coefficients. Solutions are compared to those given 
in [15]. (See [6] and [10] for corresponding problems of a concentration pulse in a porous medium flow.)

The heat transport within the water is primarily convective due to the high velocity of the water within the pipe, and it is purely conductive within the pipe walls. This suggests that our exact micro-model leads to a linear transport equation for the water in the direction parallel to its flow and a diffusion equation in the pipe wall. Although the thermal conductivity of stationary water is approximately $0.599 \frac{\mathrm{W}}{\mathrm{mK}}$ [19], the flux induced by mechanical dispersion (which accounts for the increased transverse heat flow [5]) and the advective flux lead to an effective conductivity of the water that is substantially larger than the stationary value. This effective water conductivity is essential for the construction of our exact micro-model. However, we will see on the macroscale that the diffusion term in the water parallel to the flow is still negligible in comparison with the dominant linear transport and storage terms. We shall assume that the pipe material has a conductivity that is low compared to the effective conductivity value of water. Examples we have in mind include pipes made of cast iron $\left(k_{\text {iron }}=37.2 \frac{\mathrm{W}}{\mathrm{mK}}\right)$ or bronze $\left(k_{\text {bronze }}=57.3 \frac{\mathrm{W}}{\mathrm{mK}}\right)$. See [19] for additional material properties.

\section{The Singular Micro-Model}

To describe the structure of the pipe cross-sections, we begin by considering the reference cell, $Y \equiv\left\{y=\left[y_{1}, y_{2}\right] \in \mathbb{R}^{2}:\|y\|=\sqrt{\left(y_{1}\right)^{2}+\left(y_{2}\right)^{2}}<R\right\}$ with $R>1$. In this region, we define $Y_{1} \equiv\left\{y \in \mathbb{R}^{2}:\|y\|<1\right\}, Y_{2} \equiv\left\{y \in \mathbb{R}^{2}: 1<\|y\|<R\right\}$, and note that $\bar{Y}=\bar{Y}_{1} \cup \bar{Y}_{2}$ (see Figure $2(\mathrm{a})$ ). The boundary of $Y_{1}$ is the unit circle, denoted by $\Gamma_{11}$, and that of $Y$ is the circle of radius $R$, denoted by $\Gamma_{22}$. Thus, the boundary of $Y_{2}$ is given by $\Gamma_{2}=\Gamma_{11} \cup \Gamma_{22}$. We denote the outward unit normal vector on $\Gamma_{2}$ (which points in the direction out of $Y_{2}$ ) by $\boldsymbol{\nu}$. To account for the small radii in the pipe cross sections, we scale the radii of the reference cell $Y$ by $\varepsilon>0$. Using an $\varepsilon$ superscript to denote $\varepsilon$-dependence, we represent the water region within an arbitrary cross-section by $Y_{1}^{\varepsilon} \equiv \varepsilon Y_{1}=\left\{z=\left(z_{1}, z_{2}\right)=\varepsilon y \in \mathbb{R}^{2}: y \in Y_{1}\right\}$, and the pipe wall by $Y_{2}^{\varepsilon} \equiv \varepsilon Y_{2}=\left\{z=\varepsilon y \in \mathbb{R}^{2}: y \in Y_{2}\right\}$. The corresponding boundaries are similarly denoted by $\Gamma_{11}^{\varepsilon}, \Gamma_{22}^{\varepsilon}$, and $\Gamma_{2}^{\varepsilon}$ (see Figure $2(\mathrm{~b})$ ).

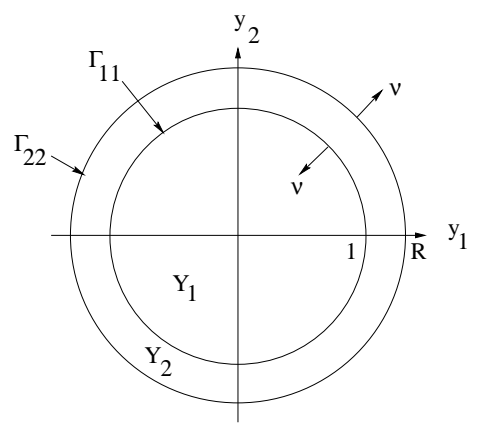

(a) Reference Cell

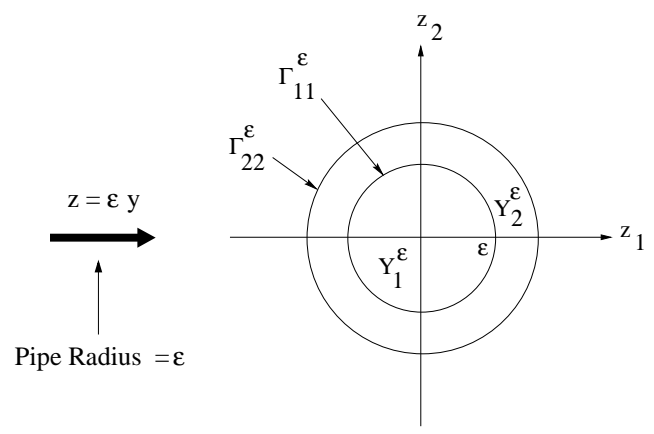

(b) Pipe cross-section

FiguRE 2. Reference cell and pipe cross-section representative. 
Let the interval $G=(0, L)$ denote the axis of the pipe (which is parallel to the water flow in region $Y_{1}^{\varepsilon}$ ), let $u_{i}^{\varepsilon}$ denote the temperature within region $Y_{i}^{\varepsilon}$ for $i=1,2$, and let the heat capacities of the water and pipe be denoted by $c_{w}$ and $c_{p}$, respectively. Furthermore, let $k_{w}$ and $\varepsilon^{2} k_{p}$ denote the conductivities of the water and pipe material. If we assume no internal heat sources or sinks and constant material properties, then the heat exchange between the water and interior pipe wall is given by the initial-boundary-value problem

$$
\begin{array}{lll}
c_{w}\left(\frac{\partial u_{1}^{\varepsilon}}{\partial t}+v(z) \frac{\partial u_{1}^{\varepsilon}}{\partial x}\right)=k_{w} \Delta u_{1}^{\varepsilon}(x, z, t), & x \in G, \quad z \in Y_{1}^{\varepsilon}, & t>0, \\
c_{p} \frac{\partial u_{2}^{\varepsilon}}{\partial t}=\varepsilon^{2} k_{p} \Delta u_{2}^{\varepsilon}(x, z, t), & x \in G, \quad z \in Y_{2}^{\varepsilon}, & t>0, \\
u_{2}^{\varepsilon}(x, z, t)=u_{1}^{\varepsilon}(x, z, t), & x \in G, \quad z \in \Gamma_{11}^{\varepsilon}, \quad t>0, \\
\varepsilon^{2} k_{p} \nabla_{z} u_{2}^{\varepsilon}(x, z, t) \cdot \boldsymbol{\nu}=k_{w} \nabla_{z} u_{1}^{\varepsilon}(x, z, t) \cdot \boldsymbol{\nu}, & x \in G, \quad z \in \Gamma_{11}^{\varepsilon}, \quad t>0, \\
k_{p} \boldsymbol{\nabla}_{\boldsymbol{z}} u_{2}^{\varepsilon}(x, z, t) \cdot \boldsymbol{\nu}=0, & x \in G, \quad z \in \Gamma_{22}^{\varepsilon}, \quad t>0, \\
u_{2}^{\varepsilon}(0, z, t)=T_{0} ; u_{2}^{\varepsilon}(L, z, t)=h_{L}(t), & & z \in Y_{2}^{\varepsilon}, \quad t>0, \\
u_{1}^{\varepsilon}(0, z, t)=T_{0} ; u_{1}^{\varepsilon}(L, z, t)=g_{L}(t), & z \in Y_{1}^{\varepsilon}, & t>0, \\
u_{1}^{\varepsilon}(x, z, 0)=u_{2}^{\varepsilon}(x, z, 0)=T_{a m b}, & x \in G, & z \in Y^{\varepsilon} .
\end{array}
$$

This is the exact micro-model. The pipe conductivity permits very high gradients in $G \times Y_{2}^{\varepsilon}$, and we will show that our choice of $\varepsilon^{2} k_{p}$ has exactly the right order of magnitude to balance the competing singularities. By rescaling the pipe cross sections with $z=\varepsilon y$, the $\varepsilon$-dependence of the geometry can be eliminated, and we obtain the singular micromodel

$$
\begin{array}{lr}
c_{w}\left(u_{1, t}^{\varepsilon}+v u_{1, x}^{\varepsilon}\right)=k_{w} u_{1, x x}^{\varepsilon}+\frac{k_{w}}{\varepsilon^{2}} \nabla_{\boldsymbol{y}} \cdot \nabla_{\boldsymbol{y}} u_{1}^{\varepsilon}+F_{1}, & x \in G, y \in Y_{1}, t>0, \\
c_{p} u_{2, t}^{\varepsilon}=\varepsilon^{2} k_{p} u_{2, x x}^{\varepsilon}+k_{p} \boldsymbol{\nabla}_{\boldsymbol{y}} \cdot \boldsymbol{\nabla}_{\boldsymbol{y}} u_{2}^{\varepsilon}+F_{2}, & x \in G, y \in Y_{2}, t>0, \\
u_{2}^{\varepsilon}(x, s, t)=u_{1}^{\varepsilon}(x, s, t), & x \in G, s \in \Gamma_{11}, t>0, \\
k_{p} \boldsymbol{\nabla}_{\boldsymbol{y}} u_{2}^{\varepsilon}(x, s, t) \cdot \boldsymbol{\nu}=\frac{k_{w}}{\varepsilon^{2}} \boldsymbol{\nabla}_{\boldsymbol{y}} u_{1}^{\varepsilon}(x, s, t) \cdot \boldsymbol{\nu}, & x \in G, s \in \Gamma_{11}, t>0, \\
k_{p} \boldsymbol{\nabla}_{\boldsymbol{y}} u_{2}^{\varepsilon}(x, s, t) \cdot \boldsymbol{\nu}=0, & x \in G, s \in \Gamma_{22}, t>0, \\
u_{2}^{\varepsilon}(0, y, t)=u_{2}^{\varepsilon}(L, y, t)=0, & y \in Y_{2}, t>0, \\
u_{1}^{\varepsilon}(0, y, t)=u_{1}^{\varepsilon}(L, y, t)=0, & y \in Y_{1}, t>0, \\
u_{i}^{\varepsilon}(x, y, 0)=T_{a m b}-w_{i}(x, 0), & i=1,2
\end{array}
$$

The homogeneous boundary conditions in (1f) and (1g) are obtained by subtracting the functions

$$
w_{1}(x, t) \equiv T_{0}\left(\frac{L-x}{L}\right)+g_{L}(t) \frac{x}{L}, \quad w_{2}(x, t) \equiv T_{0}\left(\frac{L-x}{L}\right)+h_{L}(t) \frac{x}{L}
$$


from a solution of the exact micro-model. The functions $F_{1}(x, y, t)$ and $F_{2}(x, t)$ are the result of this translation, and they are given by

$$
F_{1}(x, y, t)=\frac{c_{w} v(y)}{L}\left[T_{0}-g_{L}(t)\right]-\frac{c_{w} x}{L}\left[h_{L}(t)\right]^{\prime}, \quad F_{2}(x, t)=-\frac{c_{p} x}{L}\left[h_{L}(t)\right]^{\prime} .
$$

We note that the singular micro-model (1) is formulated on the fixed reference cell $Y$, and all of the singularities are now built into the coefficients.

We shall regard the initial-boundary-value problem (1) as a Cauchy problem in an appropriate Hilbert space, and use the theory of linear contraction semigroups to show it is well-posed for each $\varepsilon>0$. For such a development, we shall frequently refer to the resolvent problem corresponding to (1), and this is given by

$$
\begin{array}{lr}
c_{w}\left(\lambda u_{1}^{\varepsilon}+v(y) u_{1, x}^{\varepsilon}\right)=k_{w} u_{1, x x}^{\varepsilon}+\frac{k_{w}}{\varepsilon^{2}} \nabla_{\boldsymbol{y}} \cdot \nabla_{\boldsymbol{y}} u_{1}^{\varepsilon}+f_{1}(x, y), & x \in G, y \in Y_{1}, \\
c_{p} \lambda u_{2}^{\varepsilon}=\varepsilon^{2} k_{p} u_{2, x x}^{\varepsilon}+k_{p} \boldsymbol{\nabla}_{\boldsymbol{y}} \cdot \boldsymbol{\nabla}_{\boldsymbol{y}} u_{2}^{\varepsilon}+f_{2}(x, y), & x \in G, y \in Y_{2}, \\
u_{2}^{\varepsilon}(x, s)=u_{1}^{\varepsilon}(x, s), & x \in G, s \in \Gamma_{11}, \\
k_{p} \boldsymbol{\nabla}_{\boldsymbol{y}} u_{2}^{\varepsilon}(x, s) \cdot \boldsymbol{\nu}=\frac{k_{w}}{\varepsilon^{2}} \boldsymbol{\nabla}_{\boldsymbol{y}} u_{1}^{\varepsilon}(x, s) \cdot \boldsymbol{\nu}, & x \in G, s \in \Gamma_{11}, \\
k_{p} \boldsymbol{\nabla}_{\boldsymbol{y}} u_{2}^{\varepsilon}(x, s) \cdot \boldsymbol{\nu}=0, & x \in G, s \in \Gamma_{22}, \\
u_{2}^{\varepsilon}(0, y)=u_{2}^{\varepsilon}(L, y)=0, & y \in Y_{2}, \\
u_{1}^{\varepsilon}(0, y)=u_{1}^{\varepsilon}(L, y)=0, & y \in Y_{1} .
\end{array}
$$

We now show that for each $\lambda>0$ the elliptic boundary-value problem (2) is well-posed. Let us define the Hilbert space $H \equiv L^{2}\left(G \times Y_{1}\right) \times L^{2}\left(G \times Y_{2}\right)$, with the weighted scalar product

$$
(\boldsymbol{w}, \boldsymbol{\varphi})_{H}=\int_{G} \int_{Y_{1}} c_{w} w_{1}(x, y) \varphi_{1}(x, y) d y d x+\int_{G} \int_{Y_{2}} c_{p} w_{2}(x, y) \varphi_{2}(x, y) d y d x,
$$

for all $\boldsymbol{w}, \boldsymbol{\varphi}$ in $H$. For any $\boldsymbol{\varphi} \in H$, we use the notation $\boldsymbol{\varphi}=\left[\varphi_{1}, \varphi_{2}\right]$, where $\varphi_{i} \in L^{2}\left(G \times Y_{i}\right)$ for $i=1,2$. Under the above weighted $L^{2}$-scalar product, we note that $H \cong L^{2}(G \times Y)$. We also define the function space

$$
V \equiv H \cap\left\{\boldsymbol{\varphi} \in H^{1}(G \times Y): \boldsymbol{\varphi}(0, y)=\boldsymbol{\varphi}(L, y)=0, \text { a.e. } y \in Y\right\}
$$

with the norm given by $\|\varphi\|_{V}^{2}=\left\|\varphi_{1}\right\|_{H^{1}\left(G \times Y_{1}\right)}^{2}+\left\|\varphi_{2}\right\|_{H^{1}\left(G \times Y_{2}\right)}^{2}$. This is a dense subspace of $H$ and also a Hilbert space.

For any domain $W$ in $\mathbb{R}^{2}$ with smooth boundary $\partial W$, let $\gamma$ denote the usual trace map of $H^{1}(W)$ into $L^{2}(\partial W)$. We define the distributed trace map $\widetilde{\gamma}: L^{2}\left(G ; H^{1}(W)\right) \rightarrow$ $L^{2}\left(G ; L^{2}(\partial W)\right)$ by $\widetilde{\gamma} \varphi(x, s)=(\gamma \varphi(x))(s)$, and note that $\widetilde{\gamma}$ is bounded on $L^{2}\left(G ; H^{1}(W)\right)$. Implicit in the definition of $V$ is the equality of the distributed trace values (i.e., $\widetilde{\gamma} \varphi_{1}=$ $\left.\widetilde{\gamma} \varphi_{2}\right)$ across the interface $G \times \Gamma_{11}$.

Now let $\boldsymbol{\varphi} \in V$, multiply (2a) by $\varphi_{1},(2 \mathrm{~b})$ by $\varphi_{2}$, and integrate each product over $G \times Y_{1}$ and $G \times Y_{2}$, respectively. Using the internal interface and outer boundary conditions (2c)$(2 \mathrm{~g})$, and adding the resulting two integral equations, we obtain the weak formulation for the resolvent problem $(2)$,

$$
\boldsymbol{u}^{\varepsilon} \in V: \quad \lambda\left(\boldsymbol{u}^{\varepsilon}, \boldsymbol{\varphi}\right)_{H}+a^{\varepsilon}\left(\boldsymbol{u}^{\varepsilon}, \boldsymbol{\varphi}\right)=f(\boldsymbol{\varphi}), \quad \text { for all } \boldsymbol{\varphi} \text { in } V .
$$


Here, the bilinear form $a^{\varepsilon}: V \times V \longrightarrow \mathbb{R}$ is defined by

$$
\begin{aligned}
a^{\varepsilon}(\boldsymbol{w}, \boldsymbol{\varphi})=\int_{G} \int_{Y_{1}}( & \left.c_{w} v(y) w_{1, x} \varphi_{1}+k_{w} w_{1, x} \varphi_{1, x}+\frac{k_{w}}{\varepsilon^{2}} \nabla_{\boldsymbol{y}} w_{1} \cdot \nabla_{\boldsymbol{y}} \varphi_{1}\right) d y d x \\
& \quad+\int_{G} \int_{Y_{2}}\left(\varepsilon^{2} k_{p} w_{2, x} \varphi_{2, x}+k_{p} \boldsymbol{\nabla}_{\boldsymbol{y}} w_{2} \cdot \nabla_{\boldsymbol{y}} \varphi_{2}\right) d y d x, \quad \boldsymbol{w}, \boldsymbol{\varphi} \in V,
\end{aligned}
$$

and the linear functional $f: V \longrightarrow \mathbb{R}$ is given by

$$
f(\boldsymbol{\varphi})=\int_{G} \int_{Y_{1}} f_{1} \varphi_{1} d y d x+\int_{G} \int_{Y_{2}} f_{2} \varphi_{2} d y d x, \quad \boldsymbol{\varphi} \in V .
$$

From the preceding computation we see that if $\boldsymbol{u}^{\varepsilon}$ is a solution to (2), then $\boldsymbol{u}^{\varepsilon}$ satisfies (3). Conversely, starting with an appropriately smooth solution to (3), we can recover (2). This shows the equivalence between the strong formulation (2) of the resolvent problem and its weak formulation (3). The existence of a unique solution to (3) follows from Lemma 2.1 below and the Lax-Milgram theorem (see, e.g., [17], p.10).

Lemma 2.1. If $v \in L^{\infty}\left(Y_{1}\right)$, then for each $\varepsilon>0$, the bilinear form $a^{\varepsilon}(\cdot, \cdot)$ is continuous, non-negative, and $V$-elliptic: there is a constant $C_{\varepsilon}>0$ for which

$$
\left.a^{\varepsilon}(\boldsymbol{\varphi}, \boldsymbol{\varphi}) \geq C_{\varepsilon} \| \boldsymbol{\varphi}\right) \|_{V}^{2} \quad \text { for all } \boldsymbol{\varphi} \text { in } V \text {. }
$$

If we let $A^{\varepsilon}$ denote the unbounded operator determined by the triple $\left\{\mathcal{A}^{\varepsilon}, V, H\right\}$, where $\mathcal{A}^{\varepsilon} \in \mathcal{L}\left(V, V^{\prime}\right)$ is equivalent to the bilinear form $a^{\varepsilon}(\cdot, \cdot)$ on $V$, then Lemma 2.1 implies $A^{\varepsilon}$ is regular $m$-accretive on $V$. Hence, the Cauchy problem corresponding to (2) is wellposed, and the dynamics of the unique solution is governed by an analytic semigroup. That is, the singular micro-model (1) is a parabolic problem with strong regularizing effects $([11],[16],[17])$.

\section{The Distributed Microstructure Model}

For each $\varepsilon>0$, let $\boldsymbol{u}^{\varepsilon}$ be the solution to (3). If we substitute $\boldsymbol{\varphi}=\boldsymbol{u}^{\varepsilon}$ into (3), then we obtain

$$
\begin{aligned}
\lambda\left\|\boldsymbol{u}^{\varepsilon}\right\|_{H}^{2} & +k_{w}\left\|u_{1, x}^{\varepsilon}\right\|_{L^{2}\left(G \times Y_{1}\right)}^{2}+k_{w}\left\|\frac{1}{\varepsilon} \nabla_{\boldsymbol{y}} u_{1}^{\varepsilon}\right\|_{L^{2}\left(G \times Y_{1}\right)}^{2} \\
& +k_{p}\left\|\varepsilon u_{2, x}^{\varepsilon}\right\|_{L^{2}\left(G \times Y_{2}\right)}^{2}+k_{p}\left\|\nabla_{\boldsymbol{y}} u_{2}^{\varepsilon}\right\|_{L^{2}\left(G \times Y_{2}\right)}^{2} \leq\|f\|_{H^{\prime}}\left\|\boldsymbol{u}^{\varepsilon}\right\|_{H},
\end{aligned}
$$

and this a priori estimate leads to the following lemma.

Lemma 3.1. If the functions $\left\{\boldsymbol{u}^{\varepsilon}\right\}$ in $V$ are solutions to (3), then the sequences $\left\{\boldsymbol{u}^{\varepsilon}\right\}$, $\left\{u_{1, x}^{\varepsilon}\right\}$ and $\left\{\frac{1}{\varepsilon} \boldsymbol{\nabla}_{\boldsymbol{y}} u_{1}^{\varepsilon}\right\}$, as well as, $\left\{\varepsilon u_{2, x}^{\varepsilon}\right\}$ and $\left\{\boldsymbol{\nabla}_{\boldsymbol{y}} u_{2}^{\varepsilon}\right\}$, are bounded in $H, L^{2}\left(G \times Y_{1}\right)$, and $L^{2}\left(G \times Y_{2}\right)$, respectively.

This implies the existence of a subsequence $\left\{\boldsymbol{u}^{\varepsilon_{j}}\right\}$ for which each of these sequences is weakly convergent in their respective space. In addition, it shows that $\left\|\boldsymbol{\nabla}_{\boldsymbol{y}} u_{1}^{\varepsilon_{j}}\right\|_{L^{2}\left(G \times Y_{1}\right)}=$ $\varepsilon_{j}\left\|\frac{1}{\varepsilon_{j}} \nabla_{\boldsymbol{y}} u_{1}^{\varepsilon_{j}}\right\|_{L^{2}\left(G \times Y_{1}\right)} \rightarrow 0$ as $\varepsilon_{j} \rightarrow 0$, so the weak limit of $u_{1}^{\varepsilon_{j}}$ is a function of $x$ only, and then

$$
\varepsilon_{j}^{2} u_{2, x}^{\varepsilon_{j}} \rightarrow 0 \quad \text { and } \quad \nabla_{\boldsymbol{y}} u_{2}^{\varepsilon_{j}} \rightarrow \nabla_{\boldsymbol{y}} U \text { as } \varepsilon_{j} \rightarrow 0 \text {. }
$$


This leads to the function space

$$
\begin{aligned}
& V_{*} \equiv\left\{\boldsymbol{\varphi}=\left[\varphi_{1}, \varphi_{2}\right] \in H_{0}^{1}(G) \times L^{2}\left(G, H^{1}\left(Y_{2}\right)\right):\right. \\
& \gamma \varphi_{1}(x)=\gamma \varphi_{2}(x, \cdot) \text { on } \Gamma_{11}, \text { a.e. } x \in G, \\
& \text { and } \left.\varphi_{2}(0, y)=\varphi_{2}(L, y)=0, \text { a.e. } y \in Y_{2}\right\},
\end{aligned}
$$

with norm given by $\|\varphi\|_{V_{*}}^{2} \equiv\left\|\varphi_{1}\right\|_{H^{1}(G)}^{2}+\left\|\varphi_{2}\right\|_{L^{2}\left(G ; H^{1}\left(Y_{2}\right)\right)}^{2}$. If we denote the weak limit of $\left\{\boldsymbol{u}^{\varepsilon_{j}}\right\}$ in $H$ by $\boldsymbol{u}$, then this limit satisfies

$$
\boldsymbol{u}=[u, U] \in V_{*}: \quad(\lambda \boldsymbol{u}, \boldsymbol{\varphi})_{H}+a(\boldsymbol{u}, \boldsymbol{\varphi})=f(\boldsymbol{\varphi}) \text { for all } \boldsymbol{\varphi}=[\boldsymbol{\varphi}, \Phi] \in V_{*},
$$

where the bilinear form $a(\cdot, \cdot): V_{*} \times V_{*} \rightarrow \mathbb{R}$ is defined by

$$
\begin{aligned}
a(\boldsymbol{w}, \boldsymbol{\varphi}) \equiv c_{w} \int_{Y_{1}} v(y) d y \int_{G} w_{1, x}(x) \varphi_{1}(x) & d x+k_{w}\left|Y_{1}\right| \int_{G} w_{1, x}(x) \varphi_{1, x}(x) d x \\
& +\int_{G} \int_{Y_{2}} k_{p} \boldsymbol{\nabla}_{\boldsymbol{y}} w_{2}(x, y) \cdot \nabla_{\boldsymbol{y}} \varphi_{2}(x, y) d y d x
\end{aligned}
$$

Lemma 3.2. If $v \in L^{\infty}\left(Y_{1}\right)$, then the bilinear form $a(\cdot, \cdot)$ is continuous, non-negative, and $V_{*}$-elliptic: there exists a constant $C>0$ such that

$$
a(\boldsymbol{\varphi}, \boldsymbol{\varphi}) \geq C\|\boldsymbol{\varphi}\|_{V_{*}}^{2} \quad \text { for all } \boldsymbol{\varphi} \text { in } V_{*} .
$$

The ellipticity condition given in this lemma relies upon the Poincare inequality; see [16] (Theorm 5.3, p.75). The Lax-Milgram Theorem now shows that there is exactly one solution of the problem (5). By the uniqueness of this solution, and by the uniqueness of weak limits, it follows that the entire sequence $\left\{\boldsymbol{u}^{\varepsilon}\right\}$ converges weakly to $\boldsymbol{u}$ in $H$.

Lemma 3.2 implies that the corresponding unbounded operator $A$ is regular $m$-accretive on $V_{*}$, and this shows the Cauchy problem corresponding to the resolvent equation (5) is well-posed. As before, the corresponding dynamic problem is parabolic and has strong regularizing effects.

Lemma 3.3. The sequence $\left\{\boldsymbol{u}^{\varepsilon}\right\}$ converges strongly to $\{\boldsymbol{u}\}$ in $H$.

Proof. Given the weakly convergent subsequence $\left\{\boldsymbol{u}^{\varepsilon}\right\}$, let us apply $\boldsymbol{u}^{\varepsilon}-\boldsymbol{u}$ to (3). Using the linearity of the $H$-scalar product and $a^{\varepsilon}(\cdot, \cdot)$, we see that

$$
\begin{aligned}
\lambda\left(\left(\boldsymbol{u}^{\varepsilon}-\boldsymbol{u}\right), \boldsymbol{\varphi}\right)_{H} & +a^{\varepsilon}\left(\boldsymbol{u}^{\varepsilon}-\boldsymbol{u}, \boldsymbol{\varphi}\right) \\
& =f(\boldsymbol{\varphi})-\left[(\lambda \boldsymbol{u}, \boldsymbol{\varphi})_{H}+a(\boldsymbol{u}, \boldsymbol{\varphi})\right]-\varepsilon^{2} k_{p} \int_{G} \int_{Y_{2}} U_{x} \varphi_{2, x} d y d x .
\end{aligned}
$$

If we substitute $\varphi_{1}=u_{1}^{\varepsilon}(x, y)-u(x)$ and $\varphi_{2}=u_{2}^{\varepsilon}(x, y)-U(x, y)$ into the above equation, then we obtain

$$
\begin{aligned}
\lambda\left\|\boldsymbol{u}^{\varepsilon}-\boldsymbol{u}\right\|_{H}^{2} & +a^{\varepsilon}\left(\boldsymbol{u}^{\varepsilon}-\boldsymbol{u}, \boldsymbol{u}^{\varepsilon}-\boldsymbol{u}\right) \\
& =f\left(\boldsymbol{u}^{\varepsilon}-\boldsymbol{u}\right)-\left[\left(\lambda \boldsymbol{u}, \boldsymbol{u}^{\varepsilon}-\boldsymbol{u}\right)_{H}+a\left(\boldsymbol{u}, \boldsymbol{u}^{\varepsilon}-\boldsymbol{u}\right)\right] \\
& -\varepsilon^{2} k_{p} \int_{G_{Y_{2}}} \int_{U_{x}}\left(u_{2, x}^{\varepsilon}-U_{x}\right) d y d x,
\end{aligned}
$$

for every $f$ in $H$. From the weak convergence of $\left\{\boldsymbol{u}^{\varepsilon}\right\}$, the right side of $(7)$ converges to zero as $\varepsilon \rightarrow 0$. Thus, each term on the left side of (7) converges to zero. It follows that 
the sequence $\left\{\boldsymbol{u}^{\varepsilon}\right\}$ converges strongly to $\boldsymbol{u}$ in $H$. In addition, $\left\{u_{1, x}^{\varepsilon}\right\}$ converges strongly to $u_{x}$ in $L^{2}\left(G \times Y_{1}\right)$, and $\left\{\boldsymbol{\nabla}_{\boldsymbol{y}} \boldsymbol{u}^{\varepsilon}\right\}$ converges strongly to $\boldsymbol{\nabla}_{\boldsymbol{y}} \boldsymbol{u}$ in $L^{2}\left(G \times Y_{2}\right)$.

To determine the strong form of the resolvent problem $(2)$, begin by choosing $\varphi=[\varphi, \Phi]$ in $V_{*}$ for which $\varphi(x)=0$ for a.e. $x$ in $G$ and $\Phi$ is in $C_{0}^{\infty}\left(G \times Y_{2}\right)$. Then (5) yields

$$
\int_{G} \int_{Y_{2}}\left(\lambda c_{p} U \Phi+k_{p} \boldsymbol{\nabla}_{\boldsymbol{y}} U \cdot \nabla_{\boldsymbol{y}} \Phi\right) d y d x=\int_{G} \int_{Y_{2}} f_{2} \Phi d y d x
$$

for all $\Phi$ in $C_{0}^{\infty}\left(G \times Y_{2}\right)$, and this implies

$$
\lambda c_{p} U-k_{p} \boldsymbol{\nabla}_{\boldsymbol{y}} \cdot \boldsymbol{\nabla}_{\boldsymbol{y}} U=f_{2} .
$$

From (8), we note that $\boldsymbol{\nabla}_{\boldsymbol{y}} \cdot \boldsymbol{\nabla}_{\boldsymbol{y}} U$ belongs to $L^{2}\left(G \times Y_{2}\right)$. Upon substituting (8) into the right side of (5) and choosing $\Phi$ in $L^{2}\left(G, H^{1}\left(Y_{2}\right)\right)$, we obtain

$$
\int_{G} \int_{Y_{2}}\left(\boldsymbol{\nabla}_{\boldsymbol{y}} U \cdot \boldsymbol{\nabla}_{\boldsymbol{y}} \Phi+\Phi \boldsymbol{\nabla}_{\boldsymbol{y}} \cdot \boldsymbol{\nabla}_{\boldsymbol{y}} U\right) d y d x=0 .
$$

Green's formula reduces this last equation to

$$
\int_{G} \int_{\Gamma_{2}} \nabla_{\boldsymbol{y}} U \cdot \boldsymbol{\nu} \widetilde{\gamma} \Phi d s d x=0
$$

for all $\Phi$ in $L^{2}\left(G, H^{1}\left(Y_{2}\right)\right)$. Choose $\Phi$ such that $\widetilde{\gamma} \Phi=0$ a.e. on $G \times \Gamma_{11}$. Since $\Gamma_{2}=$ $\Gamma_{11} \cup \Gamma_{22}$, this implies $\nabla_{\boldsymbol{y}} U \cdot \boldsymbol{\nu}=0$ a.e. on $G \times \Gamma_{22}$.

Similarly, choose $\varphi$ in $C_{0}^{\infty}(G)$ and $\Phi(x, y)=\varphi(x)$ for a.e. $y$ in $Y_{2}$. Then (5) reduces to

$$
\begin{aligned}
\left|Y_{1}\right| \int_{G} \lambda c_{w} u(x) \varphi(x) d x+c_{w} \int_{Y_{1}} v(y) d y \int_{G} u_{x}(x) \varphi(x) d x \\
+k_{w}\left|Y_{1}\right| \int_{G} u_{x}(x) \varphi_{x}(x) d x+\int_{G}\left(\int_{Y_{2}} k_{p} \nabla_{\boldsymbol{y}} \cdot \nabla_{\boldsymbol{y}} U(x, y) d y\right) \varphi(x) d x \\
=\int_{G}\left(\int_{Y_{1}} f_{1}(x, y)\right) \varphi(x) d x
\end{aligned}
$$

for every $\varphi$ in $C_{0}^{\infty}(G)$, and this shows

$$
\begin{aligned}
\lambda c_{w} u(x)+c_{w}\left(\frac{1}{\left|Y_{1}\right|} \int_{Y_{1}} v(y) d y\right) & u_{x}(x)-k_{w} u_{x x}(x) \\
& +\frac{1}{\left|Y_{1}\right|} \int_{Y_{2}} k_{p} \nabla_{\boldsymbol{y}} \cdot \nabla_{\boldsymbol{y}} U(x, y) d y=\frac{1}{\left|Y_{1}\right|} \int_{Y_{1}} f_{1}(x, y) d y
\end{aligned}
$$

in the sense of distributions. Defining $\bar{V} \equiv \frac{1}{\left|Y_{1}\right|} \int_{Y_{1}} v(y) d y$ and applying Gauss' Theorem to the integral term over $Y_{2}$, we obtain

$$
\begin{aligned}
c_{w}(\lambda u(x) & \left.+\bar{V} u_{x}(x)\right)-k_{w} u_{x x}(x) \\
& +\frac{1}{\left|Y_{1}\right|} \int_{\Gamma_{11}} k_{p} \boldsymbol{\nabla}_{\boldsymbol{y}} U(x, y) \cdot \boldsymbol{\nu} d y=\frac{1}{\left|Y_{1}\right|} \int_{Y_{1}} f_{1}(x, y) d y .
\end{aligned}
$$

Implicit in (10) is the fact that $u_{x x}$ belongs to $L^{2}(G)$. Finally, the definition of $V_{*}$ yields the additional boundary conditions: $\tilde{\gamma} U=\widetilde{\gamma} u$ a.e. on $G \times \Gamma_{11}, U(0, y)=U(L, y)=$ 0 for a.e. $y$ in $Y_{2}$, and $u(0)=u(L)=0$. 
The preceding stationary results along with the Trotter-Kato theorem [11] lead to the main result of this section.

Proposition 3.4. For each $\varepsilon>0$, let $\left\{\boldsymbol{u}^{\varepsilon}(x, y, t)\right\}$ be the solution of the singular micromodel (1). Then, the limit $\lim _{\varepsilon \rightarrow 0} \boldsymbol{u}^{\varepsilon}=\boldsymbol{u} \equiv[u(x, t), U(x, y, t)]$ in $C([0, T] ; H)$ is the unique solution of the distributed microstructure model

$$
\begin{array}{ll}
c_{w}\left(u_{t}+\bar{V} u_{x}\right)=k_{w} u_{x x}-\frac{1}{\left|Y_{1}\right|} \int_{\Gamma_{11}} k_{p} \boldsymbol{\nabla}_{\boldsymbol{y}} U \cdot \boldsymbol{\nu} d s, & x \in G, \\
c_{p} U_{t}=k_{p} \boldsymbol{\nabla}_{\boldsymbol{y}} \cdot \boldsymbol{\nabla}_{\boldsymbol{y}} U, & x \in G, y \in Y_{2}, t>0, \\
U(x, s, t)=u(x, t), & x \in G, s \in \Gamma_{11}, t>0, \\
k_{p} \boldsymbol{\nabla}_{\boldsymbol{y}} U(x, s, t) \cdot \boldsymbol{\nu}=0, & x \in G, s \in \Gamma_{22}, t>0, \\
U(x, y, 0)=T_{a m b}, & x \in G, y \in Y_{2}, \\
u(0, t)=T_{0}, \quad u(L, t)=g_{L}(t), & x \in G,
\end{array}
$$

where $\bar{V} \equiv \frac{1}{\left|Y_{1}\right|} \int_{Y_{1}} v(y) d y$. Furthermore, for each $T>0$, the strong convergence $\boldsymbol{u}^{\varepsilon}(t, \cdot) \rightarrow \boldsymbol{u}(t, \cdot)$ in $H$ is uniform for $t \in[0, T]$.

The integral term in (11a) represents the rate of heat loss to the pipe wall at the crosssection $x \in G$, and it is computed using the normal component of the heat gradient in the wall. Using (11b) and (11d), one can write this integral as

$$
\int_{\Gamma_{11}} k_{p} \nabla_{y} U \cdot \boldsymbol{\nu} d s=\frac{\partial}{\partial t} \int_{Y_{2}} c_{p} U d y
$$

which shows it is also the rate at which heat is stored in the pipe at $x \in G$.

The singular term in (1a) arose from the rescaling of the geometry in the original micro-model, and the corresponding term in (1b) balances this singular term through the flux condition (1d). More specifically, our choice of $\varepsilon^{2}$ for the scale factor of the pipe conductivity $k_{p}$ has exactly the right order of magnitude to balance the competing singularities arising from the geometry of the pipe and the difference in materials. In the limit this resulted in a fully coupled model describing the heat exchange between the water and interior pipe wall.

The initial-boundary-value problem (11) is an example of a distributed microstructure model. The integral term in (11a) contains the effects of the microscale geometry of the boundary of the local cells, and the family of local problems (11b)-(11e) arise from the multiple scales associated with the problem. In general, the macroscale is specified by a global region, which we denote by a bounded domain $G$, and the microscale is specified by local cells, $G_{x}$. These local cells represent a magnified or scaled copy of the microstructure associated with each corresponding point $x \in G$. Within each local cell $G_{x}$ a partial differential equation describes the process within that cell, and a separate partial differential equation describes the global process throughout $G$. Any coupling between the macrostructure and microstructure will occur along the boundary of $G_{x}$, denoted by $\Gamma_{x}$, and it is the collection of these boundaries $\left\{\Gamma_{x}: x \in G\right\}$ that provides the interface on which the exchange takes place. Such problems arise naturally as limits by 
homogenization theory, and they are known to be well-suited for describing processes with multiple scales. For additional discussion of the application of distributed microstructure models in diffusion processes, see [10], [1], [2], [9], and [7].

\section{Kinetic Models}

The effective thermal conductivity of water $k_{w}$ was needed in the "exact" micro-model in Section 2 to account for the transverse motion of heat flow between the water and interior pipe wall. This led in Section 3 to the fully coupled distributed microstructure model (11) in which the linear transport and storage terms dominate. The longitudinal diffusion term $k_{w} u_{1, x x}$ could have been neglected in the original exact micro-model (1). The bilinear form obtained from such a system is not coercive on $V$, but one can regularize the system corresponding to (1) and take limits to obtain the same results without that term. Hereafter we shall neglect the longitudinal diffusion term $k_{w} u_{1, x x}$ in (11a).

We shall rewrite the distributed microstructure model in dimensionless form. This equivalent model provides a natural means of approximation and will be directly compared with others. Let us define the dimensionless quantities; $\tau \equiv \frac{\alpha}{t_{f}} t, \quad \xi \equiv \frac{\beta}{L} x$,

$$
T_{w}(x, t) \equiv \frac{u(x, t)-T_{a m b}}{T_{0}-T_{a m b}}, \quad \text { and } \quad T_{p}(x, y, t) \equiv \frac{U(x, y, t)-T_{a m b}}{T_{0}-T_{a m b}}
$$

where $\alpha=\beta=\left|t_{f} \frac{k_{p}}{c_{p}}\right|$. Introducing these quantities and (12) into our distributed microstructure model reduces (11) to the form

$$
\begin{aligned}
& \frac{\partial T_{w}}{\partial t}(x, t)+\frac{\partial T_{w}}{\partial x}=-\frac{c_{p}}{c_{w}} \frac{1}{\left|Y_{1}\right|} \frac{\partial}{\partial t} \int_{Y_{2}} T_{p} d y, \quad x \in G, \quad t>0, \\
& T_{w}(x, 0)=0, \quad x \in G, \\
& T_{w}(0, t)=1 \text {, } \\
& t>0, \\
& \left\{\begin{array}{lll}
\frac{\partial T_{p}}{\partial t}(x, y, t)=\nabla_{y} \cdot \nabla_{y} T_{p}, & x \in G, & y \in Y_{2}, \quad t>0, \\
T_{p}(x, s, t)=T_{w}(x, t), & x \in G, & s \in \Gamma_{11}, \quad t>0, \\
\nabla_{y} T_{p}(x, s, t) \cdot \boldsymbol{\nu}=0, & x \in G, & s \in \Gamma_{22}, \quad t>0, \\
T_{p}(x, y, 0)=0, & x \in G, & y \in Y_{2},
\end{array}\right.
\end{aligned}
$$

where $G=(0,1)$, and the normalized pipe and water temperatures are given by $T_{p}$ and $T_{w}$, respectively.

If we define the canonical local cell problem as

$$
\left\{\begin{array}{lll}
\frac{\partial}{\partial t} W(y, t)=\nabla_{y} \cdot \nabla_{y} W, & y \in Y_{2}, & t>0 \\
W(s, t)=1, & s \in \Gamma_{11}, \quad t>0 \\
\nabla_{y} W(s, t) \cdot \boldsymbol{\nu}=0, & s \in \Gamma_{22}, \quad t>0 \\
W(y, 0)=0, & y \in Y_{2}, &
\end{array}\right.
$$

then the solution to the subsystem (13d) is given by Duhamel's principle in the form

$$
T_{p}(x, y, t)=\left(T_{w}(x, \cdot) * W_{t}(y, \cdot)\right)(t),
$$


where $W_{t}$ denotes the time derivative of the solution to the local cell problem. The convolution in (14) is taken with respect to $t$. Using the method of separation of variables, we obtain the solution of the exchange cell problem as

$$
W(y, t)=1+\sum_{n=1}^{\infty} A_{n} \phi_{n}(y) T_{n}(t)
$$

where $T_{n}(t) \equiv e^{-\lambda_{n}^{2} t}, \phi_{n}(y)$ and $\lambda_{n}^{2}$ represent the orthonormal eigenfunctions and corresponding eigenvalues of the associated mixed Dirichlet-Neumann boundary-value problem in cylindrical coordinates, respectively, and $\left\{A_{n}\right\}$ denotes the Fourier coefficients of the constant function -1 in $L^{2}\left(Y_{2}\right)$.

If we use (14) and (15) in (13a), then we obtain a system equivalent to our distributed microstructure model given by

$$
\begin{aligned}
& \frac{\partial}{\partial t} T_{w}(x, t)+\frac{\partial}{\partial x} T_{w}=\sum_{n=1}^{\infty} \frac{c_{p}}{c_{w}} d_{n} \lambda_{n}^{2}\left(v_{n}(x, t)-T_{w}\right), \quad x \in G, \quad t>0, \\
& T_{w}(x, 0)=0, \quad x \in G, \\
& T_{w}(0, t)=1, \quad t>0, \\
& \begin{cases}\frac{\partial}{\partial t} v_{n}(x, t)=\lambda_{n}^{2}\left(T_{w}-v_{n}\right), & x \in G, \quad t>0, \\
v_{n}(x, 0)=0, & x \in G, \quad \text { for all } n \geq 1,\end{cases}
\end{aligned}
$$

where

$$
d_{n} \equiv \frac{\left(A_{n}\right)^{2}}{\left|Y_{1}\right|} \quad \text { and } \quad v_{n}(x, t) \equiv \lambda_{n}^{2}\left(T_{w}(x, \cdot) * T_{n}(\cdot)\right)(t)
$$

Note that (16) consists of a single partial differential equation and a diagonal system of ordinary differential equations. The approximation of the local cell problem by a sequence of ordinary differential equations was developed and implemented by M. Peszyńska in her work on systems with memory [14]. By deleting all but the first $N$ of the ordinary differential equations (16d), we obtain the classical $N^{\text {th }}$-order kinetic model in the nomenclature of U. Hornung [9]. For $N=1$ we have the two-temperature model given in [15], and corresponding models for flow in porous media are given in [13] and [6]. This identification of these classical models as special cases of (16) provides both a validation of these ad hoc models and a means to calibrate their coefficients. Similar remarks apply to the development of fissured medium equations of [4] from the corresponding distributed model of [2]; see [9].

\section{Numerical Results}

Finally, we shall use a numerical inversion scheme for the Laplace transform to investigate the effect of the differential equations in $(16 \mathrm{~d})$ on the overall solution. Let us denote the outlet temperature of the water at the end of the pipe by $T_{w}(1, t)$. If we denote the Laplace transform of $T_{w}(x, t)$ by $\widehat{T_{w}}(x, s)$, then the (formal) solution to system $(16)$ is given by $T_{w}(x, t)=\mathcal{L}^{-1}\left[\widehat{T_{w}}(x, s)\right]$, where

$$
\widehat{T_{w}}(x, s)=\frac{1}{s} \exp \left[-\left(1+\frac{c_{p}}{c_{w}} \sum_{n=1}^{\infty} \frac{d_{n} \lambda_{n}^{2}}{s+\lambda_{n}^{2}}\right) s x\right] .
$$


Given a pipe cross-section with inner radius $R_{i}=1$ and outer radius $R_{o}=1.25$, we use a finite partial sum approximation for the infinite series in (17), along with a modified Maple/Matlab program ([3], [8]), to plot the temperature profiles of the outlet water. The first four values of $\lambda_{n}^{2}$ and $d_{n}$ are

$$
\lambda_{n}^{2}=[36.031,351.9,983.58,1931.1], \quad d_{n}=[.4669, .0457, .0163, .0083] .
$$

In Figure 3, the infinite series in (16a) was approximated by its first term and the temperature profile of the outlet water was plotted for the first eigenvalue $\lambda_{1}^{2}=36.031$. For a comparison, we plotted the temperature profiles of the outlet water for the range of thermocoupling values given by Seliktar and Rorres in their two-temperature model [15]. As this figure shows, for small values of $\lambda_{1}^{2}$, the outlet water temperature $T_{w}(1, t)$ jumps from 0 to approximately $e^{-d_{1} \lambda_{1}^{2}}=.954$ at the flushing time $t_{f}=1$, then gradually increases towards the limiting value of 1 as $t \rightarrow \infty$.

As $\lambda_{1}^{2}$ increases, the jump in the outlet water temperature is seen to decrease at the

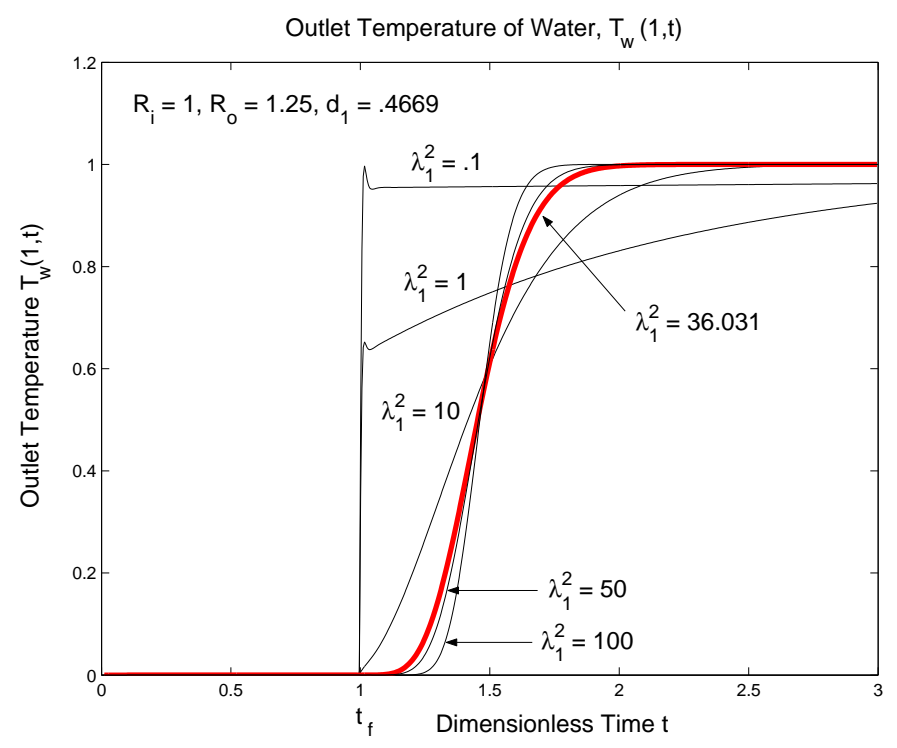

Figure 3. Comparison of Outlet Temperature Profiles.

flushing time, while a rapid increase in temperature (towards the limiting value of 1) develops at $t_{f}+d_{1}=1.4669$. This rapid increase in temperature is an approximation to the original assumption of an instantaneous jump from 0 to 1 in the inlet temperature. The coefficient $d_{1}=0.4669$ represents the time delay in the arrival of hot water due to the absorption of heat by the surrounding pipe. This comparison shows us that our firstorder kinetic model (in the nomenclature of U. Hornung [10]) is just the two-temperature model given in [15], under the specific thermal coupling value of 36.031. We also point out that $t_{f}+d_{1}=1.4669$ is the theoretical value given by the classical transport model and the method of characteristics. The graph corresponding to $\lambda_{1}^{2}=36.031$ shows that the initial "heat front" in the water travels down the pipe at a velocity slower than the actual water velocity $\bar{V}$, and its temperature profile becomes blurred as it reaches the 
end of the pipe. This is expected since the temperature difference between the water and pipe is inducing a temperature gradient in the radial direction which forces the water to lose heat to the surrounding wall as it travels down the pipe. W. Munk [13] made similar observations through a different argument. Also see [6] for a related application to the flow of a concentration pulse in a porous medium.

In Figure 4, we compare temperature profiles of the outlet water for the first-, second-, third-, and fourth-order kinetic models obtained by retaining successively more terms in (16). This figure shows that the corresponding apparent delay times in the arrival of hot water increase slightly as the partial sums increase, and the additional increase in delay time between consecutive partial sums decreases substantially. If we define hot water as $T_{w}(1, t) \geq \frac{1}{2}$, i.e., the outlet water temperature is at least one-half of the inlet water temperature, and then the wait time for hot water to emerge at the end of the pipe is at least $1.513 t_{f}$ units of time, which is approximately $\frac{1}{2} t_{f}$ units of time longer than the flushing time. Note further that for $n \geq 3, \lambda_{n}^{2}$ is relatively large, and Figure 4 suggests that an excellent approximation to system (16) (and the singular $\varepsilon$-model) is obtained by truncating the infinite series in (16) to its first two terms. The classical first-order kinetic model obtained by retaining only the first term provides a good approximation, so long as it is not used to predict the temperature value at a specific time during the emergence of the pulse. Other factors that may contribute to the delay time in the arrival of hot

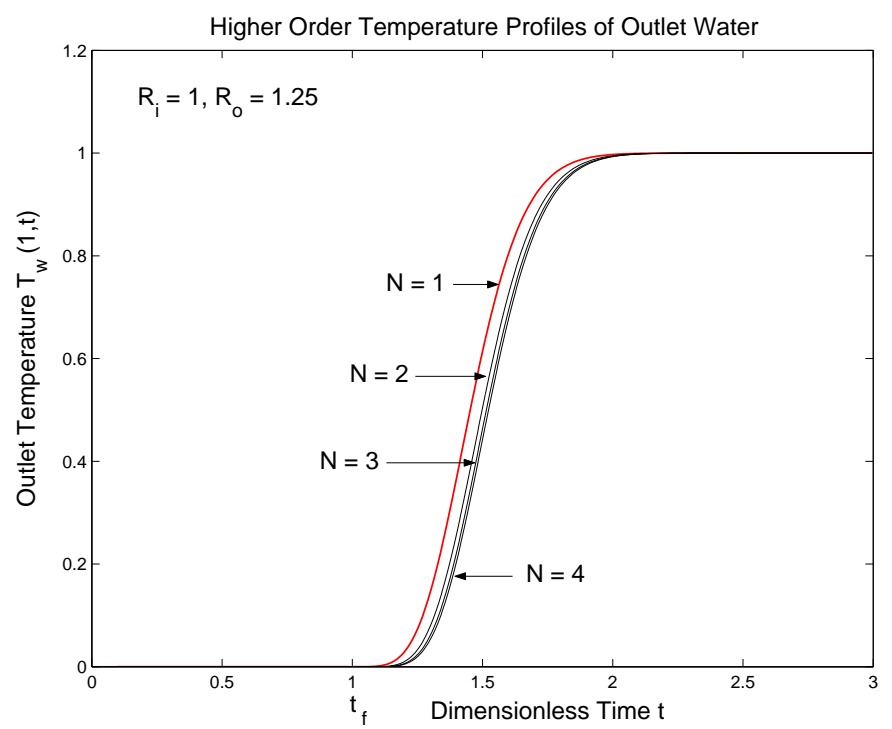

FiguRE 4. Higher Order Terms.

water at the end of the pipe are the pipe diameter, the pipe thickness, and the pipe material. In Figure 5, we plotted the temperature profiles of the outlet water for three different values of pipe thickness. As these plots suggest, an increase in pipe thickness corresponds to an increase in delay time. The other factors will have a similar effect. 


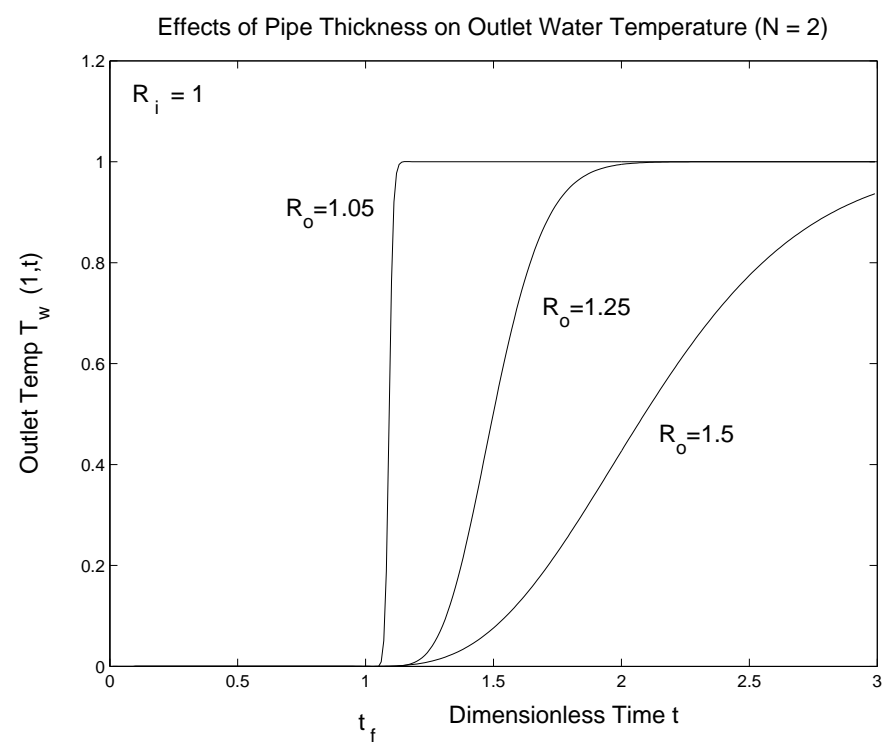

FiguRE 5. Effects of Pipe Thickness. 


\section{REFERENCES}

[1] G. Allaire, Homogenization and two-scale convergence, SIAM J. Math. Anal. 23 (1992), pp. 14821518.

[2] T. Arbogast, J. Douglas, Jr., and U. Hornung, Derivation of the double porosity model of single phase flow via homogenization theory, SIAM J. Math. Anal. 21 (1990), pp. 823-836.

[3] G.A. Articolo, Partial Differential Equations and Boundary Value Problems with Maple V, Academic Press, San Diego, (1998), pp. 169-182.

[4] G. I. Barenblatt, I. P. Zheltov, and I. N. Kochina, Basic concepts in the theory of seepage of homogeneous liquids in fissured rocks [strata], Prikl. Mat. Mekh. 24 (1960), pp. 852-864; J. Appl. Math. Mech. 24 (1960), pp. 1286-1303.

[5] J. Bear, Dynamics of Fluids in Porous media, Elsevier, New York, 1972.

[6] H.A. Deans, A mathematical model for dispersion in the direction of flow in porous media, Trans. Soc. Petr. Eng., 228 (1963), pp. 49-52.

[7] J. Douglas, Jr., M. Peszyńska, and R.E. Showalter, Single phase flow in partially fissured media, Transport in Porous Media 28 (1997), pp. 285-306.

[8] K.J. Hollenbeck, INVLAP.M: A matlab function for numerical inversion of Laplace Transforms by the de Hoog algorithm, (1998), http://www.isva.dtu.dk/staff/karl/invlap.m

[9] U. Hornung and R.E. Showalter, Diffusion models for fractured media, Jour. Math. Anal. Appl., 147 (1990), pp. 69-80.

[10] U. Hornung, Homogenization and Porous Media, Springer-Verlag, Berlin, New York, 1997.

[11] T. Kato, Perturbation Theory for Linear Operators, Springer-Verlag, Berlin Heidelberg, 1980.

[12] A. Lowan, On the Problem of the Heat Recuperator, Phil. Mag. 17 (1934), pp. 914-933.

[13] W. Munk, The Delayed Hot Water Problem, Journal of Applied Mechanics, 21 (1954), p. 193.

[14] M. Peszyńska, On a model for nonisothermal flow in fissured media, Proceedings of Differential Equations Conference, Athens, Ohio, August 3-7, 1993, Differential and Integral Equations 8 (1995), pp. 1497-1516.

[15] M. Seliktar and C. Rorres, The Flow of Hot Water From A Distant Hot-Water Tank, J. SIAM Review 36 (1994), pp. 474-479.

[16] R.E. Showalter, Hilbert Space Methods for Partial Differential Equations, Volume 1 of Monographs and Studies in Mathematics, Pitman, 1977, and Electronic Journal of Differential Equations (EJDE), Monograph 01, 1994 (available at http://ejde.math.swt.edu).

[17] R.E. Showalter, Monotone Operators in Banach Space and Nonlinear Partial Differential Equations, in Volume 49 in the series Mathematical Surveys and Monographs, American Mathematical Society, Providence, 1997.

[18] R.E. Showalter, Distributed Microstructure Models of Porous Media, International Series of Numerical Mathematics, Vol. 114, Birkhäuser Verlag Basel, 1993.

[19] F.M. White, Heat and Mass Transfer, Addison Wesley, Reading, Massachusetts, 1988. 
Department of Mathematics, University of Texas at Austin, Austin, TX 78712, USA E-mail address: show@math.utexas.edu

Department of Mathematics, University of Texas at Austin, Austin, TX 78712, USA

E-mail address: darrin@math.utexas.edu 